TRANSACTIONS OF THE

AMERICAN MATHEMATICAL SOCIETY

Volume 362, Number 7, July 2010, Pages 3779-3798

S 0002-9947(10)05040-3

Article electronically published on February 17, 2010

\title{
A NOTE ON THE VERLINDE BUNDLES ON ELLIPTIC CURVES
}

\author{
DRAGOS OPREA
}

\begin{abstract}
We study the splitting properties of the Verlinde bundles over elliptic curves. Our methods rely on the explicit description of the moduli space of semistable vector bundles on elliptic curves, and on the analysis of the symmetric powers of the Schrödinger representation of the Theta group.
\end{abstract}

\section{INTRODUCTION}

Recently, Popa defined and studied a class of vector bundles on the Jacobians of curves, which he termed the Verlinde bundles [15. The fibers of these vector bundles are the spaces of nonabelian Theta functions on the moduli spaces of bundles with fixed determinant over the curve, as the determinant varies in the Jacobian. Popa investigated the splitting properties of these bundles under certain étale pullbacks. He further used these results to prove the Strange Duality conjecture at level 1 and to study the basepointfreeness of the pluri-Theta series.

In this note, we study the Verlinde vector bundles in genus 1, correcting some of the statements of [15]. The techniques and results of our work are also useful to the understanding of the higher genus case, as we will demonstrate in [13].

To set the stage, consider a smooth complex projective curve $X$ of genus $g \geq 1$ and write $U_{X}(r, r(g-1))$ for the moduli space of rank $r$, degree $r(g-1)$ semistable bundles on $X$. This moduli space comes equipped with a canonical Theta divisor supported on the locus

$$
\Theta_{r}=\left\{V \in U_{X}(r, r(g-1)) \text {, such that } h^{0}(V)=h^{1}(V) \neq 0\right\} .
$$

Following Popa [15], we define the level $k$ Verlinde bundles on the Jacobian as the pushforwards

$$
\mathrm{E}_{r, k}=\operatorname{det}_{\star}\left(\Theta_{r}^{k}\right)
$$

under the determinant morphism

$$
\text { det }: U_{X}(r, r(g-1)) \rightarrow \operatorname{Jac}^{r(g-1)}(X) \cong \operatorname{Jac}(X) .
$$

Among the results Popa proved, we mention:

(i) the pullback of $\mathrm{E}_{r, k}$ under the multiplication morphism

$$
r: \operatorname{Jac}(X) \rightarrow \operatorname{Jac}(X)
$$

splits as a sum of line bundles;

Received by the editors September 11, 2008.

2000 Mathematics Subject Classification. Primary 14H60; Secondary 14H40.

This work was supported by NSF grant DMS-0701114. 
(ii) $\mathrm{E}_{r, k}$ is globally generated if $k \geq r+1$ and is normally generated if $k \geq 2 r+1$;

(iii) $\mathrm{E}_{r, k}$ is ample, polystable with respect to any polarization on the Jacobian, and satisfies $I T_{0}$.

In addition, it is known that the Verlinde bundles enjoy the following level-rank symmetry:

(iv) there is an isomorphism

$$
\mathrm{SD}: \mathrm{E}_{r, k}^{\vee} \cong \widehat{\mathrm{E}_{k, r}} .
$$

The hat decorating the bundle on the right hand side denotes the FourierMukai transform with kernel the normalized Poincaré bundle on the Jacobian.

The morphism (iv), sometimes termed "Strange Duality," was constructed in this form by Popa. Proofs that SD is an isomorphism can be found in [10, [3]. The case of elliptic curves, which will be relevant for us, is simpler; a discussion is contained in 7 .

To explain the results of this note, assume from now on that $X$ is a smooth complex projective curve of genus 1 . For reasons which will become clear only later, let us temporarily write $h$ for the rank of the bundles making up the moduli space. We will first show:

Theorem 1.1. Let $k, h$ and $q$ be positive integers. The Verlinde bundle $\mathrm{E}_{h, k}$ splits as a sum of line bundles iff the level $k$ is divisible by the rank $h$. When $k=h(q-1)$, we have

$$
\mathrm{E}_{h, h(q-1)} \cong \Theta^{q-1} \otimes\left(\bigoplus \mathrm{L}_{\xi}^{\oplus \mathrm{m}_{\xi}}\right) .
$$

Here, $\Theta$ is the canonical Theta bundle on the Jacobian, and the $\mathrm{L}_{\xi}$ 's are the $h$ torsion line bundles. Each line bundle $\mathrm{L}_{\xi}$ of order $\omega$ occurs with multiplicity

$$
\mathrm{m}_{\xi}=\sum_{\delta \mid h} \frac{1}{q \delta^{2}}\left(\begin{array}{c}
q \delta \\
\delta
\end{array}\right)\left\{\frac{h / \omega}{h / \delta}\right\},
$$

provided that either $h$ or $q$ is odd. If both $h$ and $q$ are even, then

$$
\mathrm{m}_{\xi}=\sum_{\delta \mid h} \frac{(-1)^{\delta}}{q \delta^{2}}\left(\begin{array}{c}
q \delta \\
\delta
\end{array}\right)\left\{\frac{h / \omega}{h / \delta}\right\} .
$$

The symbol \{\} appearing in the above statement is defined as follows. For any integer $h \geq 2$, we decompose

$$
h=p_{1}^{a_{1}} \ldots p_{n}^{a_{n}}
$$

into powers of primes. We set

$$
\left\{\frac{\lambda}{h}\right\}= \begin{cases}0 & \text { if } p_{1}^{a_{1}-1} \ldots p_{n}^{a_{n}-1} \text { does not divide } \lambda \\ \prod_{i=1}^{n}\left(\epsilon_{i}-\frac{1}{p_{i}^{2}}\right) & \text { otherwise }\end{cases}
$$

Here,

$$
\epsilon_{i}=\left\{\begin{array}{lc}
1 & \text { if } p_{i}^{a_{i}} \mid \lambda, \\
0 & \text { otherwise }
\end{array}\right.
$$

If $h=1$, the symbol is always defined to be 1 . 
Note that it was expected that the splitting of the Verlinde bundles should involve only $h$-torsion line bundles. In fact, Popa proved the isomorphism

$$
h^{\star} \mathrm{E}_{h, h(q-1)} \cong h^{\star} \Theta^{N},
$$

where $N=\frac{1}{q}\left(\begin{array}{c}h q \\ h\end{array}\right)$. However, the multiplicities $\mathrm{m}_{\xi}$ of the nontrivial bundles $\mathrm{L}_{\xi}$ were incorrectly claimed to be 0 in Propositon 2.7 of [15]. This led to an erroneous statement in Proposition 5.3. Our note corrects this oversight.

As an example, when $h$ is an odd prime, all nontrivial $h$-torsion line bundles appear in the decomposition (1.3) with the same (nonzero) multiplicity. This follows for instance by the arguments of [2, upon analyzing the action of a symplectic group on the $h$-torsion points. This is consistent with the theorem above, which specializes to

$$
\mathrm{E}_{h, h(q-1)}=\Theta^{q-1} \otimes\left(\bigoplus_{\xi \neq 0} \mathrm{~L}_{\xi}^{\oplus n} \oplus \mathcal{O}^{\oplus m}\right) .
$$

Here,

$$
n=\frac{1}{h^{2}}\left(\frac{1}{q}\left(\begin{array}{c}
q h \\
h
\end{array}\right)-1\right) \text { and } m=\frac{1}{h^{2}}\left(\frac{1}{q}\left(\begin{array}{c}
q h \\
h
\end{array}\right)-1\right)+1 .
$$

Our proof will show that

$$
m=\operatorname{dim}\left(\operatorname{Sym}^{h(q-1)} \mathrm{S}_{h}\right)^{\mathrm{H}_{h}},
$$

with $S_{h}$ being the Schrödinger representation of the Heisenberg group $H_{h}$. If $h$ is not prime, the ensuing formulas for multiplicities are more complicated, and their integrality is not immediately clear.

Theorem 1.1 is stated for the moduli spaces of bundles of degree zero. The case of arbitrary rank and degree, and of arbitrary Theta divisors will be the subject of Theorem 3.1 in Section 3.1 .

The case when the level is not divisible by the rank is slightly more involved, and requires additional ideas. We will consider this most general situation separately, in Section 3.2. To explain the final result, let us first change the notation, writing $h r$ for the rank of the bundles making up the moduli space, and letting $h k$ be the level. If $\operatorname{gcd}(r, k)=1$, then, for any $h$-torsion line bunde $\xi$, there is a unique stable bundle $\mathbf{W}_{r, k, \xi}$ on the Jacobian, having rank $r$ and determinant $\Theta^{k} \otimes \xi$. We will show:

Theorem 1.2. Assume that $\operatorname{gcd}(r, k)=1$. The Verlinde bundle of level hk splits as

$$
\mathrm{E}_{h r, h k} \cong \bigoplus_{\xi} \mathrm{W}_{r, k, \xi}^{\oplus \mathrm{m}_{\xi}} .
$$

For each h-torsion line bundle $\xi$ on the Jacobian, having order $\omega$, the multiplicity of the bundle $\mathrm{W}_{r, k, \xi}$ in the above decomposition equals

$$
\mathrm{m}_{\xi}=\sum_{\delta \mid h} \frac{(-1)^{(h+1) k r \delta}}{(r+k) \delta^{2}}\left(\begin{array}{c}
(r+k) \delta \\
r \delta
\end{array}\right)\left\{\frac{h / \omega}{h / \delta}\right\} .
$$

Finally, the methods of this work make use of the characteristic zero hypothesis. In positive characteristic, it is likely that the answer is different and that it depends on the Hasse invariant of the curve. 
After this paper was written, Fabrizio Catanese informed us that computations of similar flavor appear in [5], in connection with certain moduli spaces of complex surfaces; we would like to thank him for pointing out the reference. In this note, however, we cover most general numerics, making use of stronger representationtheoretic results for Heisenberg groups. Furthermore, we handle the combinatorics involved in the calculation differently, expressing the final answer in closed form.

\section{The PROOF OF THEOREM [1.1}

The title of this section is self-explanatory. The proof of Theorem 1.1 to be given below relies on two essential ingredients:

(i) first, the geometric input is provided by the explicit description of the moduli space of bundles over elliptic curves, as found in [1], 16];

(ii) second, it will be crucial to understand the symmetric powers of the Schrödinger representation of the Heisenberg group. An algebraic computation will determine their characters, which are related to the decomposition (1.3).

We will discuss these two items at some length in the next sections, attempting to keep the exposition reasonably self-contained. Our arguments are quite elementary, so it is plausible that some of the results below may already exist in the literature; we tried to provide references whenever possible.

2.1. Geometry. Fix an elliptic curve $(X, o)$. Throughout the paper we identify $X \cong \operatorname{Jac}^{0}(X)$ in the usual way,

$$
p \rightarrow \mathcal{O}_{X}(p-o)
$$

In [1], 16, Atiyah and Tu showed that the moduli space $U_{X}(h, 0)$ of rank $h$, degree 0 semistable vector bundles on $X$ is isomorphic to the symmetric product

$$
U_{X}(h, 0) \cong \operatorname{Sym}^{h} X .
$$

Up to $S$-equivalence, the isomorphism can be realized explicitly as

$$
\operatorname{Sym}^{h} X \ni\left(p_{1}, \ldots, p_{h}\right) \rightarrow \mathcal{O}_{X}\left(p_{1}-o\right) \oplus \ldots \oplus \mathcal{O}_{X}\left(p_{h}-o\right) \in U_{X}(h, 0) .
$$

Under these identifications, the morphism taking bundles to their determinants

$$
\operatorname{det}: U_{X}(h, 0) \rightarrow \operatorname{Jac}(X)
$$

is the Abel-Jacobi map, which in this case becomes the addition

$$
a: \operatorname{Sym}^{h} X \rightarrow X,\left(p_{1}, \ldots, p_{h}\right) \mapsto p_{1}+\ldots+p_{h} .
$$

Note that the fiber of the morphism $a$ over the point $p \in X$ is the linear series

$$
|[p]+(h-1)[o]|=|[p]-[o]+h[o]| .
$$

In fact, as an Abel-Jacobi map, the morphism $a$ has the structure of a projective bundle

$$
\mathbb{P}\left(\mathrm{V}_{h}\right) \rightarrow X
$$

where $\mathrm{V}_{h}$ is a rank $h$ vector bundle on $X$. To describe $\mathrm{V}_{h}$, we let $\mathcal{P}$ be the Poincaré bundle over $X \times X$, normalized in the usual way,

$$
\mathcal{P}=\mathcal{O}_{X \times X}(\Delta-\{o\} \times X-X \times\{o\}),
$$


with $\Delta \hookrightarrow X \times X$ being the diagonal. The Fourier-Mukai transform with kernel $\mathcal{P}$, denoted

is given by

$$
\mathbf{R S}: \mathbf{D}(X) \rightarrow \mathbf{D}(X)
$$

$$
\mathbf{R S}(\alpha)=\mathbf{R} p_{2 !}\left(p_{1}^{\star} \alpha \otimes \mathcal{P}\right)
$$

for $\alpha \in \mathbf{D}(\mathbf{X})$. Here

$$
p_{1}, p_{2}: X \times X \rightarrow X
$$

are the projections on the two factors. Using (2.2), we conclude that

$$
\mathrm{V}_{h}=\mathbf{R} \mathcal{S}\left(\mathcal{O}_{X}(h[o])\right) .
$$

A standard computation shows that $\mathrm{V}_{h}$ has rank $h$ and determinant $-[o]$; the reference 9 spells out the details. Furthermore, we note that $\mathrm{V}_{h}$ is simple since it is the Fourier-Mukai transform of a simple bundle [11. In fact, by Atiyah's classic study [1, there is a unique bundle on $X$ with all the above properties, defined inductively as the (unique) nontrivial extension

$$
0 \rightarrow \mathrm{V}_{h-1} \rightarrow \mathrm{V}_{h} \rightarrow \mathcal{O}_{X} \rightarrow 0,
$$

starting with $\mathrm{V}_{1}=\mathcal{O}_{X}(-[o])$. Alternatively, this exact sequence is obtained as the Fourier-Mukai transform of

$$
0 \rightarrow \mathcal{O}_{X}((h-1)[o]) \rightarrow \mathcal{O}_{X}(h[o]) \rightarrow \mathcal{O}_{\{o\}} \rightarrow 0 .
$$

Note that the line bundle (2.1) has a section precisely when $p_{i}=o$ for some $1 \leq i \leq h$. It follows from (1.1) that the canonical Theta divisor $\Theta_{h}$ on $U_{X}(h, 0)$ is the image of the symmetric sum

$$
[o]+\operatorname{Sym}^{h-1} X \hookrightarrow \operatorname{Sym}^{h} X .
$$

Thus, the Theta line bundle $\Theta_{h}$ agrees, at least fiberwise, with $\mathcal{O}_{\mathbb{P}\left(\vee_{h}\right)}(1)$. In fact, one can show the isomorphism

$$
\Theta_{h} \cong \mathcal{O}_{\mathbb{P}\left(\mathrm{V}_{h}\right)}(1) \text {. }
$$

Moreover, the canonical section vanishing along the Theta divisor (2.5) is the composition

$$
\mathcal{O}_{\mathbb{P}\left(\mathrm{V}_{h}\right)} \rightarrow \mathcal{O}_{\mathbb{P}\left(\mathrm{V}_{h}\right)}(1) \otimes a^{\star} \mathrm{V}_{h} \rightarrow \mathcal{O}_{\mathbb{P}\left(\mathrm{V}_{h}\right)}(1),
$$

with the second arrow given by (2.4). These observations allow us to compute the level $k$ Verlinde bundle

$$
\mathrm{E}_{h, k}=a_{\star}\left(\Theta_{h}^{k}\right)=a_{\star}\left(\mathcal{O}_{\mathbb{P}\left(\mathrm{V}_{h}\right)}(k)\right)=\operatorname{Sym}^{k} \mathrm{~V}_{h}^{\vee} .
$$

For convenience, we will write $\mathrm{W}_{h}=\mathrm{V}_{h}^{\vee}$ for the unique stable bundle on $X$ of rank $h$ and determinant $\mathcal{O}_{X}([o])$. More generally, if $\operatorname{gcd}(h, d)=1$, we let $\mathbf{W}_{h, d}$ be the unique stable bundle of rank $h$ and determinant $\mathcal{O}_{X}(d[o])$. The bundles $\mathrm{W}_{h, d}$ can be constructed inductively as successive extensions; cf. [14], page 180. Indeed, consider two consecutive terms $0 \leq \frac{d_{1}}{h_{1}}<\frac{d_{2}}{h_{2}}<1$ in the Farey sequence; i.e., assume that

$$
h_{1} d_{2}-h_{2} d_{1}=1 \text {. }
$$

Set $h=h_{1}+h_{2}, d=d_{1}+d_{2}$. Then $\mathbf{W}_{h, d}$ is the unique nontrivial extension

$$
0 \rightarrow \mathrm{W}_{h_{1}, d_{1}} \rightarrow \mathrm{W}_{h, d} \rightarrow \mathrm{W}_{h_{2}, d_{2}} \rightarrow 0 \text {. }
$$


With these preliminaries out of the way, we proceed to investigate the splitting behavior of the Verlinde bundles $\mathrm{E}_{h, k}$. Our analysis relies on the multiplicative structure of the Atiyah bundles [1, which may not be immediately obvious.

Lemma 2.1. The Verlinde bundle $\mathrm{E}_{h, k}$ splits as a sum of line bundles if and only if $h$ divides $k$.

Proof. This result will be reproved later in the paper. A more direct argument is given below. First, observe that $\mathrm{E}_{h, k}$ is a direct summand of $\mathrm{W}_{h}^{\otimes k}$. It suffices to show that these tensor powers split as sums of lines bundles iff $h$ divides $k$. In fact, something more general is true:

Claim 2.2. Assuming $\operatorname{gcd}(h, d)=1$, the tensor powers $\mathrm{W}_{h, d}^{\otimes k}$ split as sums of rank $h^{\prime}$ bundles of the form $\mathrm{W}_{h^{\prime}, d k^{\prime}} \otimes M$, where the $M$ 's are various degree 0 line bundles. Here, we set

$$
h^{\prime}=\frac{h}{\operatorname{gcd}(h, k)}, \quad k^{\prime}=\frac{k}{\operatorname{gcd}(h, k)} .
$$

To prove the Claim, we first decompose $h=h_{1} \ldots h_{s}$ into powers of primes, and pick integers $d_{1}, \ldots, d_{s}$ such that

$$
\frac{d_{1}}{h_{1}}+\ldots+\frac{d_{s}}{h_{s}}=\frac{d}{h}
$$

Then,

$$
\mathrm{W}_{h, d}=\mathrm{W}_{h_{1}, d_{1}} \otimes \ldots \otimes \mathrm{W}_{h_{s}, d_{s}} .
$$

This could be argued as follows: both sides have the same (coprime) rank and determinant, and are moreover semistable, in fact stable. Therefore, they should coincide by Atiyah's classification. With this understood, one checks that it is enough to take $h$ to be a power of a prime $p$.

For the latter case, we will need the following rephrasing of Theorems 13 and 14 in [1]. Assume $e_{1}, e_{2}, e$ are integers not divisible by $p$ and that

$$
\frac{e_{1}}{p^{a_{1}}}+\frac{e_{2}}{p^{a_{2}}}=\frac{e}{p^{a}} .
$$

Then, Atiyah showed that for certain degree 0 line bundles $M$, we have

$$
\mathrm{W}_{p^{a_{1}, e_{1}}} \otimes \mathrm{W}_{p^{a_{2}, e_{2}}}=\bigoplus_{M} \mathbf{W}_{p^{a}, e} \otimes M
$$

Thus, when $h$ is a power of a prime, the Claim follows from (2.7), by a straightforward induction on $k$.

Remark 2.3. Using a sharper version of Atiyah's results, one can prove that when $h$ is odd, the $M$ 's appearing in the Claim above are representatives for the cosets of $h$-torsion line bundles on $X$ modulo the twisting action of the group of $h^{\prime}$-torsion line bundles. The same statement should hold true for $h$ even, but Atiyah's results only show that the orders of the $M$ 's divide $2 h$. In particular, for $h$ odd and $\operatorname{gcd}(h, k)=1$, we immediately conclude that

$$
\mathrm{E}_{h, k} \cong \bigoplus_{i=1}^{m} \mathrm{~W}_{h, k},
$$

with $m=\frac{1}{h+k}\left(\begin{array}{c}h+k \\ h\end{array}\right)$. Equation (2.8) is a particular case of Theorem 1.2 
We will identify the splitting of $\mathrm{E}_{h, k}=\operatorname{Sym}^{k} \mathrm{~W}_{h}$ when the level $k$ is divisible by the rank $h$. We set

$$
q=1+\frac{k}{h}
$$

Let $\mathrm{X}_{h}$ be the group of $h$-torsion points on the elliptic curve. Let $\mathrm{G}_{h}$ be the Theta group of the line bundle $\mathcal{O}_{X}(h[o])$, which is a central extension

$$
1 \rightarrow \mathbb{C}^{\star} \rightarrow \mathrm{G}_{h} \rightarrow \mathrm{X}_{h} \rightarrow 1 \text {. }
$$

The assignment

$$
\eta \rightarrow \eta^{2 h}
$$

defines an endomorphism of $\mathrm{G}_{h}$, whose image lies in the center of $\mathrm{G}_{h}$. This is easily checked for instance using (2.9) below. Let $\mathrm{H}_{h}$ be the kernel of this endomorphism. It corresponds to an extension

$$
1 \rightarrow \mu_{2 h} \rightarrow \mathrm{H}_{h} \rightarrow \mathrm{X}_{h} \rightarrow 1
$$

where $\mu_{2 h} \hookrightarrow \mathbb{C}^{\star}$ is the group of $2 h$-roots of 1 . Finally, let $S_{h}$ denote the $h$ dimensional Schrödinger representation of $\mathrm{G}_{h}$, i.e. the unique representation such that the center of $\mathrm{G}_{h}$ acts by its natural character. We refer the reader to Mumford's paper [12] or to Chapter 6 of [4] for more details regarding Heisenberg groups and their Schrödinger representations.

Picking Theta structures, we identify $\mathrm{G}_{h}$ with the Heisenberg group

$$
\mathrm{G}_{h} \cong \mathbb{C}^{\star} \times \mathbb{Z} / h \mathbb{Z} \times \mathbb{Z} / h \mathbb{Z} .
$$

The multiplication on the right hand side is defined as

$$
(\alpha, x, y)\left(\alpha^{\prime}, x^{\prime}, y^{\prime}\right)=\left(\alpha \alpha^{\prime} \zeta^{y^{\prime} x}, x+x^{\prime}, y+y^{\prime}\right) .
$$

Here, we set

$$
\zeta=\exp \left(\frac{2 \pi i}{h}\right) .
$$

The Schrödinger representation $S_{h}$ is realized on the space of functions

$$
f: \mathbb{Z} / h \mathbb{Z} \rightarrow \mathbb{C} ;
$$

cf. [4, page 164. The action of the element $(\alpha, x, y) \in \mathrm{G}_{h}$ on a function $f$ is given by the new function

$$
F: \mathbb{Z} / h \mathbb{Z} \rightarrow \mathbb{C}, F(a)=\alpha \zeta^{y a} \cdot f(x+a) .
$$

We will first compute the pullbacks of the Verlinde bundles under the morphism $h: X \rightarrow X$ which multiplies by $h$ on the elliptic curve. Using the description of $\mathrm{V}_{h}$ as a Fourier-Mukai transform provided by (2.3), and Theorem 3.11 in [11, we obtain

$$
h^{\star} \mathrm{V}_{h} \cong \mathcal{O}_{X}(-h[o])^{\oplus h} .
$$

In fact, we claim that $\mathrm{G}_{h}$-equivariantly, we have

$$
h^{\star} \mathrm{V}_{h} \cong \mathrm{S}_{h} \otimes \mathcal{O}_{X}(-h[o]) ;
$$

see also 14, page 162. Indeed, consider the trivial bundle

$$
h^{\star} \mathrm{V}_{h} \otimes \mathcal{O}_{X}(h[o]) \cong V \otimes \mathcal{O}_{X},
$$

where $V$ is an $h$-dimensional vector space. Both factors of the tensor product on the left carry a $\mathrm{G}_{h}$-action covering the translation $\mathrm{X}_{h}$-action on the base $X$. Therefore, endowing the structure sheaf appearing on the right with the trivial $G_{h}$-action, we obtain a $\mathrm{G}_{h}$-representation on $V$. Moreover, note that the center of $\mathrm{G}_{h}$ acts 
on $V$ by homotheties. Therefore, $V \cong \mathrm{S}_{h}$, by the uniqueness of the Schrödinger representation. This establishes (2.10).

Taking determinants in (2.10), we obtain

$$
h^{\star} \mathcal{O}_{X}(-[o]) \cong \Lambda^{h} \mathrm{~S}_{h} \otimes \mathcal{O}_{X}(-h[o])^{h} .
$$

This identification is a priori only $\mathrm{G}_{h}$-equivariant, but, since the center of $\mathrm{G}_{h}$ acts trivially, the isomorphism is in fact $\mathrm{X}_{h}$-equivariant. Similarly, dualizing and taking symmetric powers in (2.10), we obtain an $\mathrm{X}_{h}$-equivariant identification

$$
h^{\star} \operatorname{Sym}^{k} \mathrm{~W}_{h} \cong \operatorname{Sym}^{k} \mathrm{~S}_{h}^{\vee} \otimes \mathcal{O}_{X}(h[o])^{k} \cong \operatorname{Sym}^{k} \mathrm{~S}_{h}^{\vee} \otimes\left(\Lambda^{h} \mathrm{~S}_{h}\right)^{q-1} \otimes h^{\star} \mathcal{O}_{X}([o])^{q-1} .
$$

Observe that the action of the central elements $\alpha$ of $G_{h}$ on the Heisenberg module

$$
\mathrm{M}_{k}=\mathrm{Sym}^{k} \mathrm{~S}_{h}^{\vee} \otimes\left(\Lambda^{h} \mathrm{~S}_{h}\right)^{q-1}
$$

is trivial, since

$$
\alpha^{-k} \cdot\left(\alpha^{h}\right)^{q-1}=1
$$

Therefore $\mathrm{M}_{k}$ is an $\mathrm{X}_{h}$-module. The $\mathrm{X}_{h}$-action splits into eigenspaces indexed by the characters $\xi$ of $\mathrm{X}_{h}$, each appearing with multiplicity $\mathrm{m}_{\xi}$ :

$$
\mathrm{M}_{k} \cong \bigoplus_{\xi} \xi^{\oplus \mathrm{m}_{\xi}}
$$

Let us write $\widehat{X}_{h}$ for the group of characters of $\mathrm{X}_{h}$. For each $\xi \in \widehat{\mathrm{X}}_{h}$, let $\mathrm{L}_{\xi}$ denote the corresponding $h$-torsion line bundle on $X$. The pullback $h^{\star} \mathrm{L}_{\xi}$ is the trivial bundle endowed with the $\mathrm{X}_{h}$-character $\xi$. Using (2.12) and (2.14), we obtain an $\mathrm{X}_{h}$-equivariant identification

$$
h^{\star} \operatorname{Sym}^{k} \mathrm{~W}_{h} \cong h^{\star}\left(\bigoplus_{\xi} \mathrm{L}_{\xi}^{\oplus \mathrm{m}_{\xi}}\right) \otimes h^{\star} \mathcal{O}_{X}([o])^{q-1} .
$$

This equivariant isomorphism determines the Verlinde bundle on the left, by general considerations about the Picard group of finite quotients. We can also give a direct argument as follows. Pushing forward the previous equation by $h$, we obtain the $\mathrm{X}_{h}$-isomorphism

$$
\operatorname{Sym}^{k} \mathrm{~W}_{h} \otimes h_{\star} \mathcal{O}_{X} \cong \bigoplus_{\xi} \mathrm{L}_{\xi}^{\oplus \mathrm{m}_{\xi}} \otimes \mathcal{O}_{X}([o])^{q-1} \otimes h_{\star} \mathcal{O}_{X}
$$

Note that $\mathrm{X}_{h}$-equivariantly,

$$
h_{\star} \mathcal{O}_{X} \cong \bigoplus_{\xi \in \widehat{\mathrm{X}}_{h}} \mathrm{~L}_{\xi}
$$

Comparing (2.15) and (2.16), and singling out the $\mathrm{X}_{h}$-invariant part, we conclude that

$$
\mathrm{E}_{h, k} \cong \operatorname{Sym}^{k} \mathrm{~W}_{h} \cong\left(\bigoplus_{\xi \in \widehat{\mathrm{X}}_{h}} \mathrm{~L}_{\xi}^{\oplus \mathrm{m}_{\xi}}\right) \otimes \mathcal{O}_{X}([o])^{q-1}
$$


2.2. Algebra. It remains to determine the multiplicities $\mathrm{m}_{\xi}$ appearing in (2.14). Regarding $\mathrm{M}_{k}$ as a representation of the finite group $\mathrm{H}_{h}$ and using the standard representation theory for finite groups as in [8, page 17], we obtain

$$
\mathrm{m}_{\xi}=\frac{1}{\left|\mathrm{H}_{h}\right|} \sum_{\eta \in \mathrm{H}_{h}} \xi\left(\eta^{-1}\right) \operatorname{Tr}_{\mathrm{M}_{k}}(\eta)
$$

We will compute this sum explicitly with the aid of the following.

Lemma 2.4. Let $\eta \in \mathrm{H}_{h}$ be an element whose image under the map $\mathrm{H}_{h} \rightarrow \mathrm{X}_{h}$ has order exactly $h / \delta$ in $\mathrm{X}_{h}$. The trace of $\eta$ on $\mathrm{M}_{k}$ equals

$$
\operatorname{Tr}_{\mathrm{M}_{k}}(\eta)=\frac{1}{q}\left(\begin{array}{c}
q \delta \\
\delta
\end{array}\right)
$$

provided that either $h$ or $q$ is odd.

Proof. We pick Theta structures, so that $\mathrm{G}_{h}$ and $\mathrm{S}_{h}$ are given by (2.9). Consider the basis $f_{1}, \ldots, f_{h}$ of $S_{h}$ given by

$$
f_{i}(j)=\delta_{i, j} .
$$

By definition, the action of

$$
\eta=(\alpha, x, y) \in \mu_{2 h} \times \mathbb{Z} / h \mathbb{Z} \times \mathbb{Z} / h \mathbb{Z}
$$

is given as

$$
\eta \cdot f_{i}=\alpha \zeta^{y(i-x)} \cdot f_{i-x} .
$$

To compute the trace of $\eta$ on $\mathrm{M}_{k}$, we may assume that $\alpha=1$, since the scaling action of the center of $\mathrm{H}_{h}$ is trivial, as remarked in (2.13).

We begin by computing the trace $\operatorname{Tr} \operatorname{Sym}^{k} \eta$ of the action of $\eta$ on $\operatorname{Sym}^{k} \mathrm{~S}_{h}^{\vee}$. For simplicity, we will first treat the case $x=0$. The eigenvalues of the action of $\eta$ on $\mathrm{S}_{h}$ are $1, \zeta_{y}, \ldots, \zeta_{y}^{h-1}$. Here, we set

$$
\zeta_{y}=\zeta^{y} .
$$

Therefore,

$$
\operatorname{Tr} \operatorname{Sym}^{k} \eta=\sum_{1 \leq i_{1} \leq \ldots \leq i_{k} \leq h} \zeta_{y}^{-\left(i_{1}+\ldots+i_{k}\right)}=\sum_{j_{1}+\ldots+j_{h}=k} \zeta_{y}^{-\left(j_{1}+2 j_{2}+\ldots+h j_{h}\right)} .
$$

In the above, $j_{r}$ denotes the number of $i$ 's which equal $r$. Now, we compute the generating series

$$
\sum_{k} \operatorname{Tr} \operatorname{Sym}^{k} \eta \cdot t^{k}=\frac{1}{1-\zeta_{y}^{-1} t} \cdot \frac{1}{1-\zeta_{y}^{-2} t} \cdots \frac{1}{1-\zeta_{y}^{-h} t} .
$$

Write

$$
h=l m,
$$

where

$$
m=\operatorname{gcd}(h, y) \text { and } \operatorname{gcd}(l, y)=1 .
$$

Then $\epsilon=\zeta_{y}$ is a primitive root of 1 of order $l$. Therefore, the product in the denominator above becomes

$$
\begin{aligned}
\left(1-\zeta_{y}^{-1} t\right) \ldots\left(1-\zeta_{y}^{-h} t\right) & =\zeta_{y}^{-h(h-1) / 2}(-1)^{h}\left((t-1)(t-\epsilon) \ldots\left(t-\epsilon^{l-1}\right)\right)^{m} \\
& =(-1)^{h+y(h-1)}\left(t^{l}-1\right)^{m}
\end{aligned}
$$


We can extract the coefficient of $t^{k}$ :

$$
\operatorname{Tr} \operatorname{Sym}^{k} \eta=(-1)^{h+y(h-1)+m+\frac{k}{l}}\left(\begin{array}{c}
-m \\
\frac{k}{l}
\end{array}\right)=(-1)^{\frac{k}{l}}\left(\begin{array}{c}
-m \\
\frac{k}{l}
\end{array}\right)=\frac{1}{q}\left(\begin{array}{c}
q m \\
m
\end{array}\right) .
$$

In particular, this computation implies that the sum (2.20) is 1 when $m=\operatorname{gcd}(h, y)$ $=1$. Moreover, the argument shows that the sum (2.20) vanishes if $k$ is not divisible by $l=\frac{h}{\operatorname{gcd}(h, y)}$.

We will now consider the $\eta$ 's in $\mathrm{H}_{h}$ for which $x \neq 0$. For these, the computation is notationally more involved. To begin, we write

$$
x=x^{\prime} s \text { and } h=h^{\prime} s,
$$

where $s=\operatorname{gcd}(h, x)$. Let $u$ be any constant with

$$
u^{h^{\prime}}=(-1)^{y x^{\prime}\left(h^{\prime}+1\right)} .
$$

Note in particular that $u^{k}=1$ for $h$ odd. For $h$ even, we have

$$
u^{k}=(-1)^{x y(q-1)} \text {. }
$$

Now, it is easy to see that the eigenvalues of $\eta$ on $S_{h}$ are

$$
\lambda_{i, j}=u \zeta_{y}^{i} \sigma^{j}, \quad 1 \leq i \leq s, 1 \leq j \leq h^{\prime},
$$

where

$$
\sigma=\exp \left(\frac{2 \pi i}{h^{\prime}}\right)
$$

In fact, we can exhibit an eigenvector for $\lambda=\lambda_{i, j}$, namely

$$
v_{\lambda}=\sum_{k=0}^{h^{\prime}-1} \lambda^{-k} \zeta_{y}^{k i-\frac{k(k+1)}{2} x} \cdot f_{i-k x}
$$

We order the indices $(i, j)$ lexicographically. The trace $\operatorname{Tr} \operatorname{Sym}^{k} \eta$ is obtained by summing all products

$$
\left(\lambda_{1, j_{1}^{1}}^{-1} \cdots \lambda_{1, j_{\bullet}^{1}}^{-1}\right)\left(\lambda_{2, j_{1}^{2}}^{-1} \cdots \lambda_{2, j_{\bullet}^{2}}^{-1}\right) \cdots\left(\lambda_{s, j_{1}^{s}}^{-1} \cdots \lambda_{s, j_{\bullet}}^{-1}\right),
$$

where

$$
1 \leq j_{1}^{i} \leq j_{2}^{i} \leq \ldots \leq j_{\bullet}^{i} \leq h^{\prime} .
$$

Let $a_{1}$ be the number of terms in the product whose first index is $1 ; a_{2}, \ldots, a_{s}$ have a similar meaning. We require $a_{1}+\ldots+a_{s}=k$. After substituting (2.23) in the product above, we sum over the $j$ 's, keeping the $a$ 's fixed. We have seen already in the derivation of (2.20) that the sum

$$
\sum_{1 \leq j_{1}^{i} \leq \cdots \leq j_{a_{i}}^{i} \leq h^{\prime}} \sigma^{-\left(j_{1}^{i}+\ldots+j_{a_{i}}^{i}\right)}
$$

is 0 if $h^{\prime}$ does not divide $a_{i}$, and it equals 1 otherwise. Therefore, writing $a_{i}=h^{\prime} a_{i}^{\prime}$, we need to evaluate

$$
\sum_{a_{1}^{\prime}+\ldots+a_{s}^{\prime}=\frac{k}{h^{\prime}}} \zeta_{y}^{-h^{\prime} a_{1}^{\prime}} \zeta_{y}^{-2 h^{\prime} a_{2}^{\prime}} \cdots \zeta_{y}^{-s h^{\prime} a_{s}^{\prime}}=\sum_{a_{1}^{\prime}+\ldots+a_{s}^{\prime}=\frac{k}{h^{\prime}}} \gamma_{y}^{-\left(a_{1}^{\prime}+\ldots+s a_{s}^{\prime}\right)} .
$$


Here, we set $\gamma=\exp \left(\frac{2 \pi i}{s}\right)$, so that $\zeta_{y}^{h^{\prime}}=\gamma_{y}$. We have already computed sums of this type in (2.20). We obtained the answer

$$
\frac{1}{q}\left(\begin{array}{c}
q \delta \\
\delta
\end{array}\right)
$$

for $\delta=\operatorname{gcd}(s, y)=\operatorname{gcd}(h, x, y)$. This expression gives the trace $\operatorname{Tr} \operatorname{Sym}^{k} \eta$ when $h$ is odd. The formula includes the previously considered case $x=0$, for which $\delta=m$. The sign change (2.22) is required when $h$ is even.

Finally, the trace of $\eta$ on $\Lambda^{h} S_{h}$ is computed using (2.19):

$$
\eta \cdot f_{1} \wedge \ldots \wedge f_{h}=(-1)^{x(h+1)} \prod_{i=1}^{h} \zeta_{y}^{i-x} \cdot f_{1} \wedge \ldots \wedge f_{h}=(-1)^{(h+1)(x+y)} f_{1} \wedge \ldots \wedge f_{h} .
$$

This completes the proof when $h$ is odd. When $h$ is even, we take into account the sign corrections of the previous paragraph and (2.22). We append formula (2.24) by the overall sign

$$
(-1)^{x y(q-1)} \cdot(-1)^{(x+y)(h+1)(q-1)}=(-1)^{(x y+x+y)(q-1)} .
$$

This does not change (2.24) when $q$ is odd, proving the lemma. When $h$ and $q$ are both even, we note, for further use, that the overall sign of (2.24) can be rewritten as

$$
(-1)^{\operatorname{gcd}(h, x, y)}=(-1)^{\delta}
$$

We proceed to calculate the sum (2.18). We claim that the multiplicity $\mathrm{m}_{\xi}$ depends only on the order of the character $\xi \in \widehat{X}_{h}$. To this end, consider the group Aut $\left(\mathrm{H}_{h}, \mu_{2 h}\right)$ of automorphisms of $\mathbf{H}_{h}$ which restrict to the identity on the center $\mu_{2 h}$. As essentially remarked in 2, the characters appearing in the $\mathrm{X}_{h^{-}}$ representation $\mathrm{M}_{k}$ are exchanged by the action of Aut $\left(\mathrm{H}_{h}, \mu_{2 h}\right)$. Beauville's argument is based on the observation that for each $F \in \operatorname{Aut}\left(\mathrm{H}_{h}, \mu_{2 h}\right)$, the standard $\mathrm{H}_{h}$-module structure of $\mathrm{S}_{h}, \rho: \mathrm{H}_{h} \rightarrow \mathrm{GL}\left(\mathrm{S}_{h}\right)$, is isomorphic to the twisted module structure $F \circ \rho: \mathrm{H}_{h} \rightarrow \mathrm{GL}\left(\mathrm{S}_{h}\right)$. This follows by examining the character of the center of $\mathrm{H}_{h}$, and by making use of the uniqueness of the Schrödinger representation. The same observation applies to the associated $\mathrm{H}_{h}$-module $\mathrm{M}_{k}$. With this understood, our claim is a consequence of the lemma below. This result is possibly known, yet for completeness we decided to include the argument. Note that the lemma is not indispensable for the proofs to follow, yet it allows for some simplification of the formulas.

Lemma 2.5. Under the action of $\mathrm{Aut}\left(\mathrm{H}_{h}, \mu_{2 h}\right)$, two characters of $\mathrm{X}_{h}$ belong to the same orbit if and only if they have the same order in $\widehat{\mathrm{X}}_{h}$.

Proof. Fix two characters $\chi_{1}, \chi_{2}$ of $\mathrm{X}_{h}$ :

$$
\chi_{i}: \mathrm{X}_{h} \rightarrow \mathbb{C}^{\star},(x, y) \rightarrow \zeta^{a_{i} x+b_{i} y}, 1 \leq i \leq 2 .
$$

The condition on the orders of $\chi_{1}$ and $\chi_{2}$ translates into

$$
\operatorname{gcd}\left(h, a_{1}, b_{1}\right)=\operatorname{gcd}\left(h, a_{2}, b_{2}\right):=\tau .
$$

This implies that we can solve the equations below, with the Greek letters as the unknowns:

$$
a_{1} \lambda+b_{1} \mu=a_{2} \quad \bmod h, a_{1} \nu+b_{1} \gamma=b_{2} \quad \bmod h .
$$


We claim that we may further achieve

$$
\lambda \gamma-\mu \nu=1 \bmod h .
$$

This can be seen for instance as follows. By the Chinese Remainder Theorem, we may take $h$ to be a power of a prime. In this case, assume first that $\tau=1$. Starting with any solution of (2.27), define a new quadruple

$$
\lambda^{\prime}=\lambda+b_{1} x, \mu^{\prime}=\mu-a_{1} x, \nu^{\prime}=\nu+b_{1} y, \gamma^{\prime}=\gamma-a_{1} y .
$$

The assumption $\tau=1$ implies that we can find a pair $(x, y)$ such that (2.28) holds:

$$
\lambda^{\prime} \gamma^{\prime}-\mu^{\prime} \nu^{\prime}=(\lambda \gamma-\mu \nu)+b_{2} x-a_{2} y \equiv 1 \quad \bmod h .
$$

For arbitrary $\tau$, after dividing by $\tau$, and using the case we already proved, we may assume that (2.27) is satisfied $\bmod h$, and that (2.28) holds true $\bmod h / \tau$. We lift the solution using Hensel's lemma, ensuring that (2.28) is also satisfied $\bmod h$.

Finally, define $F: \mathrm{H}_{h} \rightarrow \mathrm{H}_{h}$ by

$$
F(\alpha, x, y)=\left(\alpha \zeta^{\frac{1}{2}\left(\lambda \mu x^{2}+\nu \gamma y^{2}+2 \mu \nu x y\right)}, \lambda x+\nu y, \mu x+\gamma y\right) .
$$

Equation (2.28) is used to prove that $F$ is an automorphism of $\mathrm{H}_{h}$, while equation (2.27) shows that $F$ sends $\chi_{1}$ to $\chi_{2}$.

Henceforth, for the computation of (2.18), we will take $\xi$ to be the character

$$
\xi=\xi_{\lambda}: \mathbb{Z} / h \mathbb{Z} \times \mathbb{Z} / h \mathbb{Z} \ni(x, y) \mapsto \zeta_{\lambda}^{x+y}=\zeta^{\lambda(x+y)} \in \mathbb{C}^{\star} .
$$

Here, we assume that $\lambda$ divides $h$, so that the character $\xi$ has order

$$
\omega=\frac{h}{\lambda} \text {. }
$$

Assume that either $h$ or $q$ is odd. Using Lemma 2.4 we rewrite (2.18) as

$$
\mathrm{m}_{\xi}=\frac{1}{h^{2}} \sum_{\delta \mid h} \frac{1}{q}\left(\begin{array}{c}
q \delta \\
\delta
\end{array}\right)\left(\sum_{\operatorname{gcd}(h, x, y)=\delta} \xi_{\lambda}(x, y)\right) .
$$

If both $h$ and $q$ are even, each term in (2.29) is multiplied by the sign $(-1)^{\delta}$, as it follows from (2.26). In this case,

$$
\mathrm{m}_{\xi}=\frac{1}{h^{2}} \sum_{\delta \mid h} \frac{(-1)^{\delta}}{q}\left(\begin{array}{c}
q \delta \\
\delta
\end{array}\right)\left(\sum_{\operatorname{gcd}(h, x, y)=\delta} \xi_{\lambda}(x, y)\right) .
$$

We will evaluate formulas (2.29) and (2.30) in terms of the character (1.6) defined in the introduction.

Lemma 2.6. We have

$$
\sum_{\operatorname{gcd}(h, x, y)=\delta} \xi_{\lambda}(x, y)=\frac{h^{2}}{\delta^{2}}\left\{\frac{h / \omega}{h / \delta}\right\} .
$$

Proof. Replacing $h, x$ and $y$ by $h / \delta, x / \delta$ and $y / \delta$, respectively, we may assume $\delta=1$. To solve this case, let us set

$$
\mathrm{N}_{\lambda}(h)=\sum_{\operatorname{gcd}(h, x, y)=1} \xi_{\lambda}(x, y)=\sum_{\operatorname{gcd}(h, x, y)=1} \zeta_{\lambda}^{x+y} .
$$


It suffices to show that

$$
\mathrm{N}_{\lambda}(h)=h^{2}\left\{\frac{\lambda}{h}\right\}
$$

This is immediate when $h=p^{a}$ is a power of a prime. In this case, if $p^{a} \mid \lambda$, the left hand side of (2.32) counts the pairs $1 \leq x, y \leq p^{a}$ such that $\operatorname{gcd}\left(p^{a}, x, y\right)=1$. Their number is $p^{2 a-2}\left(p^{2}-1\right)$, which equals the right hand side. Otherwise, since the distinct roots of unity add up to 0 , we have

$$
\sum_{\left(x, y, p^{a}\right)=1} \zeta_{\lambda}^{x+y}=-\sum_{p \mid(x, y)} \zeta_{\lambda}^{x+y}
$$

If $p^{a-1} \mid \lambda$, then all terms in the last sum are equal to 1 , hence giving the answer $-p^{2 a-2}$. Finally, if $p^{a-1}$ does not divide $\lambda$, then replacing $\zeta_{\lambda}$ by $\zeta_{p \lambda}$, we sum all distinct roots of unity of order $p^{a-1} / \operatorname{gcd}\left(p^{a-1}, \lambda\right)$, each appearing with equal multiplicity. This gives the answer 0 .

The general case follows by induction on the number of prime factors of $h$, once we establish the multiplicativity in $h$ of the function $\mathrm{N}_{\lambda}(h)$. Let $h=h_{1} h_{2}$ with $\operatorname{gcd}\left(h_{1}, h_{2}\right)=1$. Choose integers $u, v$ such that

$$
h_{1} u+h_{2} v=1 .
$$

By the Chinese Remainder Theorem, the pairs $(x, y) \bmod h$ are in one-to-one correspondence with the pairs $\left(x_{1}, y_{1}\right) \bmod h_{1},\left(x_{2}, y_{2}\right) \bmod h_{2}$ such that

$$
\begin{array}{ccc}
x \equiv x_{1} & \bmod h_{1}, x \equiv x_{2} & \bmod h_{2}, \\
y \equiv y_{1} & \bmod h_{1}, y \equiv y_{2} & \bmod h_{2} .
\end{array}
$$

Explicitly, we have

$$
x=h_{1} u x_{2}+h_{2} v x_{1} \quad \bmod h, y=h_{1} u y_{2}+h_{2} v y_{1} \quad \bmod h .
$$

The condition $\operatorname{gcd}(h, x, y)=1$ is equivalent to

$$
\operatorname{gcd}\left(h_{1}, x_{1}, y_{1}\right)=1, \operatorname{gcd}\left(h_{2}, x_{2}, y_{2}\right)=1 \text {. }
$$

We compute

$$
\begin{aligned}
\mathrm{N}_{\lambda}(h) & =\sum_{\operatorname{gcd}(h, x, y)=1} \zeta_{\lambda}^{x+y}=\sum_{\operatorname{gcd}\left(h_{1}, x_{1}, y_{1}\right)=1, \operatorname{gcd}\left(h_{2}, x_{2}, y_{2}\right)=1} \zeta_{\lambda}^{h_{2} v\left(x_{1}+y_{1}\right)} \cdot \zeta_{\lambda}^{h_{1} u\left(x_{2}+y_{2}\right)} \\
& =\mathrm{N}_{\lambda v}\left(h_{1}\right) \mathrm{N}_{\lambda u}\left(h_{2}\right)=h_{1}^{2}\left\{\frac{\lambda v}{h_{1}}\right\} \cdot h_{2}^{2}\left\{\frac{\lambda u}{h_{2}}\right\}=h^{2}\left\{\frac{\lambda}{h_{1}}\right\}\left\{\frac{\lambda}{h_{2}}\right\}=h^{2}\left\{\frac{\lambda}{h}\right\} .
\end{aligned}
$$

In the last line, we used the fact that the factors $u$ and $v$ do not change the symbol \{\} since these numbers are prime to $h_{2}$ and $h_{1}$, respectively.

Putting together (2.17), (2.29), (2.30) and Lemma 2.6 we complete the proof of Theorem 1.1 .

\section{Arbitrary NUMERICS}

3.1. Arbitrary rank and degree. We will now discuss a variant of Theorem 1.1. which covers the case of arbitrary rank and degree. Let $r, d$ be two integers with

$$
h=\operatorname{gcd}(r, d) \text {. }
$$

Write

$$
r=h r^{\prime}, d=h d^{\prime}, \text { where } \operatorname{gcd}\left(r^{\prime}, d^{\prime}\right)=1 \text {. }
$$


We will consider Theta divisors on the moduli space $U_{X}(r, d)$. Their definition requires the choice of a twisting vector bundle $N$ of complementary slope

$$
\mu(N)=-\frac{d}{r}
$$

We set

$$
\Theta_{r, N}=\left\{V \in U_{X}(r, d), \text { such that } h^{0}(V \otimes N)=h^{1}(V \otimes N) \neq 0\right\} .
$$

To avoid confusion, even though it may be notationally cumbersome, we decorate the Theta's by the twisting bundles $N$, and by the rank of the bundles in the moduli space.

It is convenient to assume that $N$ has the minimal possible rank $r^{\prime}$. The level $k$ Verlinde bundle

$$
\mathrm{E}_{r, k}^{N}=\operatorname{det}_{\star}\left(\Theta_{r, N}^{k}\right)
$$

is obtained by pushing forward the pluri-Theta bundle $\Theta_{N}^{k}$ on $U_{X}(r, d)$ via the morphism

$$
\operatorname{det}: U_{X}(r, d) \rightarrow \operatorname{Jac}^{d}(X) \text {. }
$$

As before, we have an isomorphism

$$
U_{X}(r, d) \cong \operatorname{Sym}^{h} X .
$$

Set-theoretically, this isomorphism is essentially defined by twisting (2.1) by the unique indecomposable vector bundle $\mathrm{W}_{r^{\prime}, d^{\prime}}$ of rank $r^{\prime}$ and determinant $d^{\prime}[o]$ on $X$. More precisely, if $\left(p_{1}, \ldots, p_{h}\right)$ are $h$ points of $X$, pick $\left(q_{1}, \ldots, q_{h}\right)$ such that

$$
r^{\prime} \cdot q_{i}=p_{i}, 1 \leq i \leq h .
$$

Then, the isomorphism (3.2) is given by

$\operatorname{Sym}^{h} X \ni\left(p_{1}, \ldots, p_{h}\right) \mapsto \mathrm{W}_{r^{\prime}, d^{\prime}} \otimes \mathcal{O}_{X}\left(q_{1}-o\right) \oplus \ldots \oplus \mathbf{W}_{r^{\prime}, d^{\prime}} \otimes \mathcal{O}_{X}\left(q_{h}-o\right) \in U_{X}(r, d)$.

Note that the answer on the right hand side of (3.3) is independent of the choice of $q_{i}$. Indeed, any two $q_{i}$ 's must differ by an $r^{\prime}$-torsion point $\chi$. However, by Atiyah's classification,

$$
\mathrm{W}_{r^{\prime}, d^{\prime}} \otimes \mathrm{L}_{\chi} \cong \mathrm{W}_{r^{\prime}, d^{\prime}},
$$

as both bundles are simple, of the same rank and determinant. It was observed in [16, and it is clear from (3.3), that the determinant

$$
\operatorname{det}: U_{X}(r, d) \rightarrow \operatorname{Jac}^{d}(X)
$$

becomes the addition morphism

$$
a: \operatorname{Sym}^{h} X \rightarrow X,\left(p_{1}, \ldots, p_{h}\right) \rightarrow p_{1}+\ldots+p_{h} .
$$

Here, we used the identification

$$
X \cong \operatorname{Jac}(X) \cong \operatorname{Jac}^{d}(X)
$$

with the second arrow given by twisting degree zero line bundles by $\mathcal{O}_{X}(d[o])$. Via this identification, the divisor $\Theta_{1, \mathcal{O}(-d[o])}$ on $\operatorname{Jac}^{d}(X)$ corresponds to the canonical Theta on $\operatorname{Jac}(X)$. 
Finally, we can easily identify the Theta divisors on $U_{X}(r, d)$. There is a natural choice for the twisting bundle $N$, namely the Atiyah bundle $N_{0}=\mathrm{W}_{r^{\prime},-d^{\prime}}$. It was shown in [1], and it follows from equation (3.4), that the tensor product

$$
\mathrm{W}_{r^{\prime},-d^{\prime}} \otimes \mathrm{W}_{r^{\prime}, d^{\prime}}=\bigoplus_{\chi} \mathrm{L}_{\chi}
$$

splits as the direct sum of all $r^{\prime}$-torsion line bundles $\mathrm{L}_{\chi}$. As a consequence of (3.1), (3.3), (3.5), we see that for the bundles $V$ in the Theta divisor, we have $q_{i}=\chi$ for some $r^{\prime}$-torsion point $\chi$, and some $1 \leq i \leq h$. Thus, $\Theta_{r, N_{0}}$ is the image of the symmetric sum

$$
[o]+\operatorname{Sym}^{h-1} X \hookrightarrow \operatorname{Sym}^{h} X .
$$

We have therefore recovered (2.5), and thus reduced the computation to the case we already studied.

Theorem 3.1. Fix $r$ and $d$ to be two integers with $h=\operatorname{gcd}(r, d)$, and let $N$ be a vector bundle of slope

$$
\mu(N)=-\frac{d}{r}
$$

and of minimal rank. Then, $\mathrm{E}_{r, k}^{N}$ splits as a sum of line bundles iff $h$ divides $k$. If $k=h(q-1)$, then

$$
\mathrm{E}_{r, k}^{N} \cong\left(\Theta_{1,(\operatorname{det} N)^{h}}\right)^{q-1} \otimes\left(\bigoplus_{\xi \in \widehat{\mathrm{X}}_{h}} \mathrm{~L}_{\xi}^{\oplus \mathrm{m}_{\xi}}\right) .
$$

Here the $\mathrm{m}_{\xi}$ are given by the same formulas (1.4) and (1.5) as in Theorem 1.1.

Proof. When $N_{0}=\mathrm{W}_{r^{\prime},-d^{\prime}}$, the statement is a consequence of the above discussion and the proof of Theorem 1.1. The general case follows from here, since both the Verlinde bundle and the right hand side only change by translations. To see this, set

$$
L=\operatorname{det} N \otimes\left(\operatorname{det} N_{0}\right)^{-1} .
$$

On the one hand, formulas of Drezet-Narasimhan [6] imply that

$$
\mathrm{E}_{r, k}^{N}=\operatorname{det}_{\star}\left(\Theta_{r, N}^{k}\right)=\operatorname{det}_{\star}\left(\Theta_{r, N_{0}} \otimes \operatorname{det}^{\star} L\right)^{k}=\mathrm{E}_{r, k}^{N_{0}} \otimes L^{k} .
$$

In the above, we view the degree 0 line bundle $L$ on $X$ as a line bundle on the Jacobian in the standard way. On the other hand, we have

$$
\Theta_{1,(\operatorname{det} N)^{h}}=\Theta_{1,\left(\operatorname{det} N_{0}\right)^{h}} \otimes L^{h} .
$$

The theorem follows by putting these observations together.

3.2. Arbitrary level and rank. In this subsection we will prove Theorem 1.2. We will keep the same notation as in the introduction, writing $h r$ for the rank, and letting $h k$ be the level, with $\operatorname{gcd}(r, k)=1$. We will determine the splitting type of the Verlinde bundle

$$
\mathrm{E}_{h r, h k}=\operatorname{det}_{\star}\left(\Theta_{h r}^{h k}\right)=\operatorname{Sym}^{h k} \mathrm{~W}_{h r},
$$

obtained by pushing forward tensor powers of the canonical Theta bundle $\Theta_{h r}$ via

$$
\operatorname{det}: U_{X}(h r, 0) \rightarrow \operatorname{Jac}(X) \cong X .
$$

The case of nonzero degree and arbitrary Theta's is entirely similar, and we will leave the details to the interested reader. 
Proof of Theorem 1.2. We first consider the case when $r$ is odd. The arguments used to prove Theorem 1.1 go through with only minor changes. It suffices to check that the decomposition (1.7),

$$
\mathrm{E}_{h r, h k} \cong \bigoplus_{\xi} \mathrm{W}_{r, k, \xi}^{\mathrm{m}_{\xi}}
$$

holds $\mathrm{X}_{h r}$-equivariantly, after pullback by the morphism $h r$. The pullback of the left hand side is evaluated $\mathrm{G}_{h r}$-equivariantly via (2.12):

$$
(h r)^{\star} \mathrm{E}_{h r, h k} \cong(h r)^{\star} \operatorname{Sym}^{h k} \mathrm{~W}_{h r} \cong \mathcal{O}_{X}(h r[o])^{h k} \otimes \operatorname{Sym}^{h k} \mathrm{~S}_{h k}^{\vee} .
$$

For the right hand side, recall first that $\mathrm{W}_{r, k, \xi}$ has rank $r$ and determinant $\mathcal{O}_{X}(k[o])$ $\otimes \xi$. By comparing ranks and degrees, we see that

$$
\mathrm{W}_{r, k, \xi} \cong \mathrm{W}_{r, k} \otimes \mathrm{L}_{\chi} \text {. }
$$

Here $\mathrm{L}_{\chi}$ is any $h r$-torsion line bundle with $\mathrm{L}_{\chi}^{r}=\mathrm{L}_{\xi}$. Note that $\chi$ is uniquely defined only up to $r$-torsion line bundles. The ambiguity inherent in the choice of $\chi$ will be shown to be inessential later. Observe that the pullback $(h r)^{\star} \mathrm{L}_{\chi}$ is the trivial bundle, endowed with the $\mathrm{X}_{h r}$-character $\chi$.

We will determine the pullback of $\mathrm{W}_{r, k}$ by the morphism $h r$. As a first step, we show that nonequivariantly

$$
r^{\star} \mathrm{W}_{r, k} \cong \mathcal{O}_{X}(k r[o])^{\oplus r} .
$$

The ingredients needed for the proof of (3.8) are found in Lemma 22 of Atiyah's paper [1. There, it is explained that all indecomposable factors of $r^{\star} \mathrm{W}_{r, k}$ have the same rank $r^{\prime}$ and degree $k^{\prime}$. Therefore,

$$
r^{\star} \mathrm{W}_{r, k} \cong \bigoplus_{i=1}^{r / r^{\prime}} \mathrm{W}_{r^{\prime}, k^{\prime}} \otimes M_{i}
$$

for some line bundles $M_{i}$. In fact, examining Atiyah's arguments, one can prove a little bit more. Using (3.5), we observe that

$$
r^{\star} \mathrm{W}_{r, k} \otimes r^{\star} \mathrm{W}_{r, k}^{\vee} \cong \bigoplus_{1}^{r^{2}} \mathcal{O}_{X} .
$$

The above tensor product contains $\mathrm{W}_{r^{\prime}, k^{\prime}} \otimes \mathrm{W}_{r^{\prime}, k^{\prime}}^{\vee} \otimes M_{i} \otimes M_{j}^{-1}$ as a direct summand, for any $i$ and $j$. Now, applying equation (3.5) again, we see that

$$
\mathrm{W}_{r^{\prime}, k^{\prime}} \otimes \mathrm{W}_{r^{\prime}, k^{\prime}}^{\vee} \cong \bigoplus_{\rho} L_{\rho}
$$

the sum being taken over the $r^{\prime}$-torsion points $\rho$. This clearly gives a contradiction, unless $r^{\prime}=1$ and the bundles $M_{i}$ and $M_{j}$ coincide. In conclusion, we proved that

$$
r^{\star} \mathrm{W}_{r, k} \cong \bigoplus_{i=1}^{r} M,
$$

for a suitable line bundle $M$. Taking determinants we obtain that

$$
M \cong \mathcal{O}_{X}(k r[o]) \otimes P,
$$


for some $r$-torsion line bundle $P$. We claim that $P$ is symmetric, i.e. $(-1)^{\star} P \cong P$. When $r$ is odd, these two facts together imply that $P$ must be trivial, proving (3.8). The symmetry of $P$ is a consequence of (3.9) and of the symmetry of $\mathrm{W}_{r, k}$. Indeed,

$$
(-1)^{\star} \mathrm{W}_{r, k} \cong \mathrm{W}_{r, k}
$$

as both bundles are simple, and have the same rank and determinant.

Having established (3.8), we compute

$$
(h r)^{\star} \mathrm{W}_{r, k} \cong \mathcal{O}_{X}(h r[o])^{h k} \otimes R,
$$

where $R$ is an $r$-dimensional vector space. In fact, $R$ carries a representation of the Theta group $\mathrm{G}_{h r}$, such that the center acts with weight $-h k$. However, this does not determine the representation $R$ uniquely, not even as a representation of $\mathrm{H}_{h r}$. In fact, one can show that there are precisely $h^{2}$ representations $R_{i, j}$ of $\mathrm{H}_{h r}$ with central weight $-h k$ [17; they will be indexed by integers $i, j \in \mathbb{Z} / h \mathbb{Z} \times \mathbb{Z} / h \mathbb{Z}$.

To determine $R$, we will use the following commutative diagram:

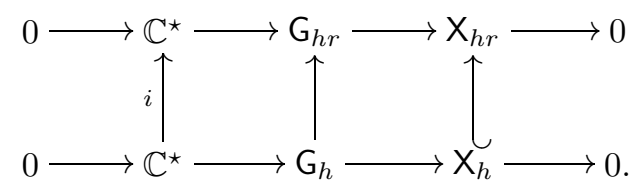

Here, the morphism $i$ is the $r$-fold cover $\alpha \mapsto \alpha^{r}$, and the middle arrow is the natural morphism of Theta groups $\mathrm{G}_{h} \rightarrow \mathrm{G}_{h r}$. Via this diagram, we may consider the action of the group $G_{h}$ on both sides of (3.10). Recall from equation (2.11) that $\mathrm{G}_{h}$-equivariantly, we have

$$
\mathcal{O}_{X}(h r[o])^{h k} \cong \mathcal{O}_{X}(h[o])^{h k r} \cong h^{\star} \mathcal{O}_{X}([o])^{k r} \otimes\left(\Lambda^{h} \mathrm{~S}_{h}\right)^{k r} .
$$

Therefore, using (3.10), we see that $\mathrm{G}_{h}$-equivariantly,

$$
R \otimes\left(\Lambda^{h} \mathrm{~S}_{h}\right)^{k r}=h^{\star}\left(r^{\star} \mathrm{W}_{r, k} \otimes \mathcal{O}_{X}(-k r[o])\right) .
$$

Note that the left hand side is an $\mathrm{X}_{h}$-module, since the center of $\mathrm{G}_{h}$ acts trivially; to this end, recall that the morphism $i$ is an $r$-fold covering of the centers. By equation (3.8), the right hand side is the pullback of a trivial vector bundle, carrying a trivial $\mathrm{X}_{h}$-action. Consequently, the $\mathrm{X}_{h}$-representation $R \otimes\left(\Lambda^{h} \mathrm{~S}_{h}\right)^{k r}$ is trivial.

This latter observation pins down the $\mathrm{H}_{h r}$-representation $R$. Let us again pick Theta structures, identifying the Theta group $\mathrm{H}_{h r}$ with the Heisenberg group. The characters of the $h^{2}$ representations $R_{i, j}$ were computed in Theorem 3 in [17. There it was proved that the trace of $\eta=(\alpha, x, y) \in \mathrm{H}_{h r} \cong \mu_{2 h r} \times \mathbb{Z} / h r \mathbb{Z} \times \mathbb{Z} / h r \mathbb{Z}$ equals

$$
\operatorname{Trace}_{R_{i, j}}(\eta)= \begin{cases}r \alpha^{-h k} \zeta^{i x+j y} & \text { if }(x, y) \in \mathrm{X}_{h}, \text { i.e. if }(x, y) \in r \mathbb{Z} / h r \mathbb{Z} \times r \mathbb{Z} / h r \mathbb{Z}, \\ 0 & \text { otherwise. }\end{cases}
$$

Here $\zeta=\exp \left(\frac{2 \pi i}{h r}\right)$. The character of the $\mathrm{H}_{h^{-}}$-representation $\left(\Lambda^{h} \mathrm{~S}_{h}\right)^{k r}$ was calculated in (2.25):

$$
\text { Trace }(\eta)=\alpha^{h k r}(-1)^{(h+1)(x+y) k r} \text {. }
$$


Since $R \otimes\left(\Lambda^{h} \mathrm{~S}_{h}\right)^{k r}$ is a trivial $\mathrm{X}_{h}$-module, we must have $i=j=\frac{h r k(h+1)}{2}$. Then, the trace of $R$ becomes

$$
\operatorname{Trace}_{R}(\eta)= \begin{cases}r \alpha^{-h k}(-1)^{(h+1) k r(x+y)} & \text { if }(x, y) \in \mathrm{X}_{h}, \\ 0 & \text { otherwise. }\end{cases}
$$

Making use of (3.6) and (3.8), we can now check that both sides of (1.7) agree equivariantly after pullback by $h r$. It remains to prove that $\mathrm{H}_{h r}$-equivariantly:

$$
\operatorname{Sym}^{h k} \mathrm{~S}_{h r}^{\vee} \cong R \otimes \bigoplus_{\chi} \chi^{\oplus \mathrm{m}_{\chi}} .
$$

In this sum, the $\chi$ 's are $h^{2}$ representatives of the characters of $\mathrm{X}_{h r}$, modulo those characters of $\mathrm{X}_{h r}$ which restrict trivially to the subgroup $\mathrm{X}_{h} \hookrightarrow \mathrm{X}_{h r}$. Taking representatives is necessary to avoid repetitions. Indeed, by comparing characters, we see that

$$
R \otimes \chi \cong R
$$

iff $\chi$ restricts trivially to the subgroup $\mathrm{X}_{h}$. This equation also takes care of the ambiguity seemingly present in the pullback of (3.7) by $h r$. Note moreover that each representative $\chi$ appearing in the sum (3.13) restricts to a well-defined character $\xi$ of $\mathrm{X}_{h}$, hence giving rise to an $h$-torsion line bundle $\mathrm{L}_{\xi}$ on $X$. We will write $\omega$ for the order of this line bundle.

In (3.13), the multiplicities $\mathrm{m}_{\chi}$ are claimed to have the expressions given in equation (1.8) of the theorem. Checking (3.13) amounts to a character calculation. For the left hand side, the character was essentially computed in Lemma 2.4 Going through the proof of the lemma, we see that the trace of $\eta=(\alpha, x, y) \in \mathrm{H}_{h r}$ on $\operatorname{Sym}^{h k} \mathrm{~S}_{h r}^{\vee}$ is zero, unless $(x, y)$ is an $h$-torsion point, say of order $h / \delta$ in $\mathrm{X}_{h}$. In the latter case,

$$
\text { Trace }(\eta)=(-1)^{x y k(h+1)} \alpha^{-h k} \cdot \frac{r}{r+k}\left(\begin{array}{c}
(r+k) \delta \\
r \delta
\end{array}\right) .
$$

It suffices to check that the formula

$$
\mathrm{m}_{\chi}=\frac{1}{2 h^{3} r} \sum_{\eta=(\alpha, x, y) \in \mu_{2 h r} \times \mathrm{X}_{h}} \operatorname{Trace}_{\operatorname{Sym}^{h k} \mathrm{~S}_{h r}}(\eta) \cdot \operatorname{Trace}_{R}(\eta)^{-1} \cdot \chi(\eta)^{-1}
$$

yields the same answer as (1.8). Substituting (3.12) and (3.14), and recalling that $\xi$ denotes the restriction of $\chi$ to $\mathrm{X}_{h}$, we obtain

$$
\mathrm{m}_{\chi}=\frac{1}{h^{2}} \sum_{\delta \mid h} \frac{(-1)^{(h+1) k r \delta}}{r+k}\left(\begin{array}{c}
(r+k) \delta \\
r \delta
\end{array}\right) \sum_{(x, y) \text { has order } h / \delta} \xi(x, y) .
$$

By Lemma 2.6, this expression can be rewritten as

$$
\mathrm{m}_{\chi}=\sum_{\delta \mid h} \frac{(-1)^{(h+1) k r \delta}}{(r+k) \delta^{2}}\left(\begin{array}{c}
(r+k) \delta \\
r \delta
\end{array}\right)\left\{\frac{h / \omega}{h / \delta}\right\} .
$$

This completes the proof when $r$ is odd.

When $r$ is even, $k$ must be odd, since $\operatorname{gcd}(r, k)=1$. Therefore, the theorem holds true for the Verlinde bundle $\mathrm{E}_{h k, h r}$. We will now use the level-rank symmetry of the Verlinde bundles under the Fourier-Mukai transform

$$
\mathrm{E}_{h r, h k}^{\vee} \cong \widehat{\mathrm{E}_{h k, h r}},
$$


which was explained in item (iv) of the introduction. We claim that the Atiyah bundles enjoy the analogous symmetry under Fourier-Mukai:

$$
\mathrm{W}_{r, k, \xi}^{\vee} \cong \widehat{\mathrm{W}_{k, r, \xi}} \text {. }
$$

Indeed, the case of trivial $\xi$ is the following well-known isomorphism generalizing (2.3):

$$
\mathrm{W}_{r, k}^{\vee} \cong \widehat{\mathrm{W}_{r, k}}
$$

This is a consequence of the fact that both bundles are simple, of the same rank, and same determinant; alternatively, one may argue using the construction of the Atiyah bundles as successive extensions, explained in Section 2.1. The case of general $\xi$ is an immediate corollary, since the bundles involved differ only by translations. To see this, pick any line bundle $M$ with $M^{k}=\xi$, and let $\tau_{M}$ denote the translation induced by $M$ on the elliptic curve. We compute

$$
\widehat{\mathrm{W}_{k, r, \xi}} \cong \widehat{\mathrm{W}_{k, r} \otimes} M \cong \tau_{M}^{\star} \widehat{\mathrm{W}_{k, r}} \cong \tau_{M}^{\star} W_{r, k}^{\vee} \cong \mathrm{W}_{r, k, \xi}^{\vee}
$$

The first and last isomorphism follow as usual by Atiyah's classification, while the second is a general fact about the Fourier-Mukai transform [11.

We conclude the proof of the theorem by collecting the above observations.

\section{ACKNOWLEDGEMENTS}

We would like to thank Alina Marian for conversations related to this topic and for many useful suggestions, and Mihnea Popa for e-mail correspondence.

\section{REFERENCES}

1. M. Atiyah, Vector bundles over an elliptic curve, Proc. London Math. Soc., 7 (1957), 414-452. MR 0131423 (24:A1274)

2. A. Beauville, The Cobble hypersurfaces, C. R. Math. Acad. Sci. Paris, 337 (2003), no. 3, 189-194. MR2001133 (2004k:14056)

3. P. Belkale, The strange duality conjecture for generic curves, J. Amer. Math. Soc. 21 (2008), 235-258. MR2350055 (2009c:14059)

4. C. Birkenhake, H. Lange, Complex abelian varieties, Springer-Verlag, Berlin-New York, 1992. MR 1217487 (94j:14001)

5. F. Catanese, C. Ciliberto, Symmetric products of elliptic curves and surfaces of general type, J. of Alg. Geom. 2 (1993), 389-411. MR.1211993 (94i:14040)

6. J. M. Drezet, M.S. Narasimhan, Groupe de Picard des variétés de modules de fibrés semistables sur les courbes algébriques, Invent. Math. 97 (1989), no. 1, 53-94. MR999313 (90d:14008)

7. R. Donagi, L. Tu, Theta functions for $\mathrm{SL}(n)$ versus $\mathrm{GL}(n)$, Math. Res. Lett. 1 (1994), no. 3, 345-357. MR1302649 (95j:14012)

8. J. Harris, W. Fulton, Representation Theory, Springer-Verlag, Berlin-New York, 1991. MR.1153249 (93a:20069)

9. G. Hein, D. Ploog, Stable bundles and Fourier-Mukai transforms on elliptic curves, Contributions to Algebra and Geometry 46 (2005), 423-434. MR2196927(2007a:14038)

10. A. Marian, D. Oprea, The level-rank duality for non-abelian theta functions, Invent. Math. 168 (2007), no. 2, 225-247. MR2289865 (2007k:14070)

11. S. Mukai, Duality between $D(X)$ and $D(X)$ with its application to Picard sheaves, Nagoya Math. J. 81 (1981), 153-175. MR607081 (82f:14036)

12. D. Mumford, On equations defining abelian varieties I, Inventiones Math. 1 (1966), 287-354. MR0204427 (34:4269)

13. D. Oprea, The Verlinde bundles and the semihomogeneous Wirtinger duality, submitted, arXiv: 0831.4962.

14. A. Polishchuk, Abelian varieties, theta functions and the Fourier-Mukai transform, Cambridge University Press, Cambridge, 2003. MR.1987784 (2004m:14094) 
15. M. Popa, Verlinde bundles and generalized theta linear series, Trans. Amer. Math. Soc., 354 (2002), no. 5, 1869-1898. MR.1881021 (2003i:14046)

16. L. Tu, Semistable bundles over an elliptic curve, Adv. Math 98 (1993), no. 1, 1-26. MR.1212625 (94a:14008)

17. J. Schulte, Harmonic analysis on finite Heisenberg groups, European J. Combin. 25 (2004), no. 3, 327-338. MR2036470(2005e:43009)

Department of Mathematics, University of California, San Diego, 9500 Gilman Drive, La Jolla, CALifornia 92093

E-mail address: doprea@math.ucsd.edu 